\title{
Searches for Squarks and Gluinos with ATLAS
}

\author{
Oliver Ricken, on behalf of the ATLAS collaboration ${ }^{1, \star}$ \\ ${ }^{1}$ Physikaliches Institut, Universität Bonn, Nussallee 12, 53115 Bonn, Germany
}

\begin{abstract}
One of the most versatile and attractive extensions to the successful yet incomplete Standard Model of particle physics is Supersymmetry - a theory the ATLAS experiment at the Large Hadron Collider is looking for in its recorded data. Due to the nature of proton-proton collisions, the recorded physics events are mainly produced via the strong force. This fact makes searches for the superpartners of the gluon and the quarks particularly promising.

This document provides an overview of searches for squarks and gluinos using the ATLAS experiment and describes two of the major analyses in detail. The analysis strategies are outlined, the results discussed and interpreted. Finally, an outlook onto other searches for strongly produced Supersymmetry with ATLAS is given.
\end{abstract}

\section{Introduction}

Being able to describe most phenomena observed in experimental particle physics, the Standard Model still leaves several open questions on both sides of particle physics, theoretical and experimental alike.

As an extended frame into which the Standard Model can be embedded, Supersymmetry (SUSY, for a pedagogical introduction see [1]) has been proposed as a theoretical model that is able to accommodate for many shortcomings of the Standard Model but for which experimental evidence is still to be found. This document reports on the latest results in the search for strong production of Supersymmetry using the ATLAS experiment [2] at the Large Hadron Collider LHC [3].

To illustrate the different approaches in the search for Supersymmetry with ATLAS, two different analyses following different strategies and searching for different models of Supersymmetry are introduced. After presenting the theoretical models being searched for, the analysis strategies and the obtained results are shown and discussed. In a brief outlook, further searches for strong production of SUSY are outlined.

\section{Supersymmetry and the Standard Model}

The most complete and successful theory in particle physics that is experimentally most thoroughly tested and confirmed is the Standard Model. It is able to describe interactions between elementary particles using three of the four fundamental forces of nature - gravity is not considered. This ability lets the Standard Model explain the formation of matter, the production and decay of heavier particles,

\footnotetext{
${ }^{\star}$ e-mail: oliver.ricken@cern.ch
} 
and the origin of the fundamental particles' properties. All in all, the Standard Model has been able to provide answers to most questions that arose from observed phenomena in the past and to predict observations before they were made, rendering itself a successful theory of particle physics. However, the successful model is yet neither complete nor able to explain all currently verified observations. Among other phenomena which the Standard Model is not able to describe is the existence of dark matter. Moreover, it is unable to predict the measured value of the Higgs boson mass in a way that does not exploit large, unnatural corrections - a problem referred to as the hierarchy problem. As a logical consequence of these flaws of the model, extensions to it and larger models to embed it into have been proposed, the most prominent being the theory of Supersymmetry (SUSY) [1].

As the name suggests, SUSY brings a new symmetry to the world of particle physics. In this larger model — of which the Standard Model would be a low-energy limit—a new fundamental symmetry between fermions and bosons is introduced. The Standard Model fermions receive bosonic superpartners while the bosons are accompanied by new fermions. These new particles allow, among many other effects, for cancellation of the huge corrections to the Higgs boson's mass introduced by couplings to Standard Model fermions, hence solving the aforementioned hierarchy problem.

If SUSY were to exist in the manner as described so far, the new particles were much likely already discovered - a bosonic electron-doppelgänger would have been visible in many experiments, for example-which is not the case until today. As a consequence, SUSY has got to be a broken symmetry. Since the minimal way of parametrising SUSY still needs more than 100 parameters ${ }^{1}$, many approaches to restrict the available parameter space by making different assumptions and proposing different mechanisms of symmetry breaking have lead to a variety of different models of SUSY, none of them being found any evidene for as of now. As a consequence and due to the fact that nature is not expected to prefer one designated breaking mechanism of SUSY over others, the search strategies of particle physics experiments have changed.

In order to find a SUSY signature regardless of any particular model and to be able to cover as many different models when interpreting the results, Simplified Models are being looked for nowadays. These models usually contain one particular SUSY signature: only one process produces a fixed set of sparticles that, in turn, decay with fixed branching ratios to a set of stable and/or detectable particles. Simplified Models are not described by parameters that arise from the breaking mechanism of a designated theory but rather by pseudo-observables such as the masses of the sparticles involved. Depending on the outcome of an experimental analysis of such a model, either the discovery of a New Physics's signature can be claimed or limits on the describing parameters can be set - within the boundaries of the underlying assumptions and constraints of such a Simplified Model, of course. For model-builders on the theory side of particle physics, Simplified Models can help to narrow down the variety of prosperous new models to look for and are hence particularly worth searching for.

\section{SUSY Searches at ATLAS - Strategies \& Approaches}

As outlined in section 2, the landscape of models of SUSY and Simplified Models is rich and not all of them are well testable at a collider such as the LHC. Due to the fact that the LHC collides protons, interactions driven by the strong force are more abundant than electro-weak processes. As a consequence, models producing gluinos and squarks via the strong interaction are expected to be well accessible and hence studied in various fashions. The ATLAS collaboration pursues two major approaches here:

- Analyses look for rather generic signatures of beyond-Standard-Model physics that are easily produced by the strong interaction to cover as much ground as possible in as many parts of the SUSY

\footnotetext{
${ }^{1}$ The Minimal Supersymmetric Standard Model comprises 104 parameters [1]
} 


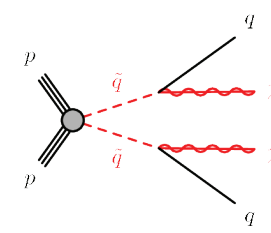

(a)

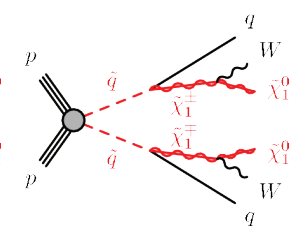

(b)

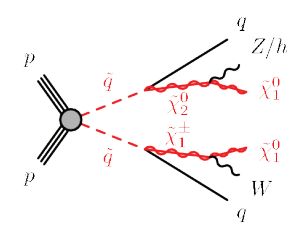

(c)

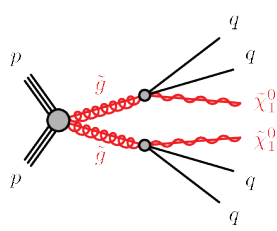

(d)

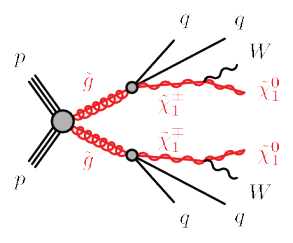

(e)

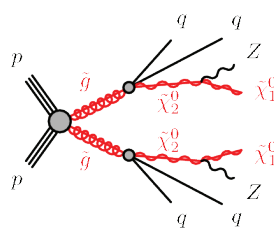

(f)

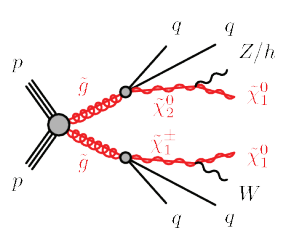

(g)

Figure 1. Signal diagrams of the Simplified Models studied in [4], covering gluino and squark production.

phase space as possible. An example for such an analysis is the search for multiple jets and missing transverse energy along with the absence of any lepton ${ }^{2}$ as it is presented in [4] and described in more detail in section 4 .

- An alternative approach in the search for SUSY is to look for the production of the sparticles which are expected to be the easiest to find. Due to the nature of SUSY, the superpartners of the Standard Model's third generation fermions - the heaviest fermions - are expected to be the lightest sparticles and hence rather abundantly produced at lower energies. The expectation is thus to observe the presence of top and bottom squarks along with tau sleptons first. Searches tailored towards signatures of such third generation sparticles are another work horse of ATLAS. An example is the search for pair production of top squarks using signatures of two leptons as presented in [5] and described in section 5. The corresponding analyses and results by the CMS collaboration can be found in [6] and [7], and [8], respectively.

\section{The Hunt for Gluinos and Squarks - Multiple Jets, Missing Energy and the Absence of Leptons as a Signature}

The way an analysis in the search for physics beyond the Standard Model is designed strongly depends on the model(s) searched for. In the case of the search for signatures with multiple jets, missing transverse energy and the absence of leptons, the Simplified Models [4] searched for are models of direct squark ${ }^{3}$ or gluino pair production via the strong force. After the production step, different decay chains are considered as can be inferred from Figure 1, all of them having in common the presence of multiple jets (here, originating from quarks), missing energy (introduced by the undetected Lightest Supersymmetric Particle (LSP), the neutralino) and the absence of any leptons. The last requirement limits the possible decays of $W$ and $Z$ bosons to pure hadronic ones.

\footnotetext{
${ }^{2}$ The term 'lepton' refers to electrons and muons exclusively. Since the ATLAS detector can only distinguish tau leptons from electrons and muons if they decay hadronically, taus are treated and referred to individually.

${ }^{3}$ In this context, the term 'squark' refers to the superpartners of first and second generation quarks. This terminology is chosen to separate these searches from endeavours to find third generation sparticles. Moreover, in the models investigated here all squarks regardless of their flavour and chirality are degenerate in mass.
} 


\subsection{Analysis Strategy}

The approach chosen to access the rare and strongly suppressed SUSY signal in this analysis is to separate the available phase space into different parts to control and validate the predictions of Standard Model background and to extract the expected signal:

- Signal Regions (SR): As the name suggests, SRs are designed to ensure the presence of the signal searched for in an analysis. In order to be able to separate the desired signal from the potentially present Standard Model background, SRs are usually designed in such a way that they suppress any Standard Model process as strongly as possible. Design in this context refers to imposing selection criteria (cuts) that cut away Standard Model events and leave signal events mostly unaffected, leading to the desired separation effect. Having a signal-dominated region of phase space, extraction of results and their statistical interpretation can be addressed.

- Control Regions (CR): The aim of CRs is to isolate designated Standard Model backgrounds by imposing selection criteria that foster their presence and suppress other backgrounds and any signal of interest. Once a CR has reached the desired purity for one background, a fit of the prediction (usually Monte-Carlo simulations (MC), sometimes data-driven templates) to the actually observed data can be carried out. This way, an estimate of the fitted background is best-tailored to the observed data and expected to be reliable even in different parts of phase space (c. f. next point). It is important that CRs (for different backgrounds) do not overlap in phase space, neither among each other, nor with VRs (details see below) or SRs - a property referred to as orthogonality. Orthogonality is often achieved by inverting cuts between regions. Only then double-counting of events in the fits can be avoided to provide an unbiased result. A typical approach in an analysis like this one is to have CRs for all major backgrounds expected to contribute to the analysis.

- Validation Regions (VR): In contrast to CRs, VRs are not used for fitting background predictions to observed data but to study the quality of those very fits. To not overlap with the CRs and to probe the quality of extrapolation in variables defining SRs, VRs are usually located in the parts of phase space in between CRs and SRs. If the agreement between background and observed data in the SR-defining variables is good, the prediction is deemed trustworthy to be used in the SRs. In order to not be biased by signal contamination, VRs are sought to be signal-free - despite their vicinity to the SRs.

This analysis designs a total of 24 SRs probing different parts of model phase space, different models (c. f. Figure 1), and using different selection approaches - while here the $m_{\mathrm{eff}}$-based approach is presented, the so-called 'Recursive Jigsaw Approach' is explained in detail in [4]. Depending on the SR their estimation is being used for, different CRs are used to obtain the correct normalisation of the contributing Standard Model backgrounds. Common to all sets of CRs are CRs for the processes $W+$ Jets, Top, $Z \rightarrow v v+$ Jets, and QCD Multijet events. While $W+$ Jets and Top are estimated using the corresponding MC predictions directly, $Z \rightarrow v v+$ Jets is estimated by fitting $\gamma+$ Jets events and applying the obtained fit result to genuine $Z \rightarrow v v+$ Jets in the SRs afterwards. This approach is justified by the difficulty to obtain a $Z \rightarrow v v+$ Jets dominated CR and the similarities between $Z \rightarrow v v$ and $\gamma$-based signatures in the detector. The contribution of Multijet events arising from QCD is estimated in a semi-data-driven way by using the so-called 'Jet-Smearing'-method [4].

The results of an exemplary set of CRs-after application of the scaling factors obtained by fitting the normalisation of the Standard Model prediction in those very CRs to the observed data-are shown in Figure 2. The red arrows indicate the cut value which is chosen to define the actual CR where the fit is carried out. Beyond those arrows, the larger abundance of the background to which each $\mathrm{CR}$ is tailored with respect to the remaining other Standard Model contributions is visible. This way, confidence in the obtained background normalisations is ensured. 


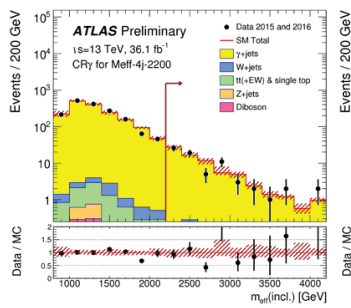

(a) $\gamma+$ Jets CR

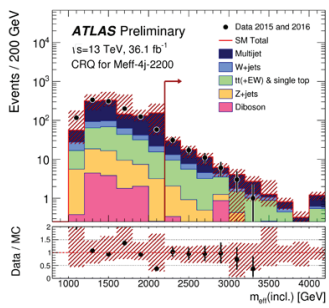

(b) Multijet CR

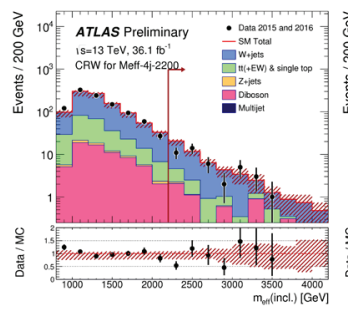

(c) $W+$ Jets CR

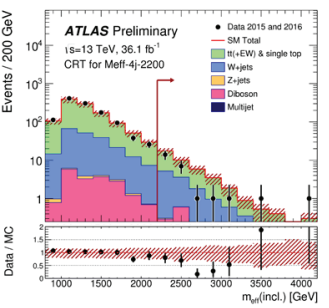

(d) Top CR

Figure 2. Distributions of the main discriminating variable $m_{\mathrm{eff}}$ before being cut on to define the set of control regions of one specific signal region. Such sets of CRs are designed for all SRs. The red arrow indicates the cut value that defines the final $\mathrm{CR}$ in which the fit of the background is performed. After application of that selection criterion, the CRs are rich in the background they are intended to probe. The distributions are shown after application of the normalisations obtained in the fit. The red lines indicate the total Standard Model prediction, while the red hatched band represents its total uncertainty (one standard deviation of combined statistical and systematic uncertainties). The last bin in each distribution contains the overflow bin of the histogram. The lower panels show the bin-wise ratios of data/MC with accordingly propagated uncertainties [4].

\subsection{Analysis Results \& Interpretations}

The results of interest in such an analysis primarily lie in the signal regions. When applying the obtained fit results from the CRs to the considered backgrounds and overlaying the signal expectations, the SRs can be studied. An exemplary SR distribution is depicted in Figure 3(a); again, the red arrow indicates the cut value used to obtain the actual SR.

Summarising all 24 SRs of this analysis, Figure 3(b) shows the obtained yields for data and the predicted backgrounds integrated into one bin for each SR. The bottom plot shows the deviation between observed data and predicted background as a ratio with accordingly normalised uncertainty bands: looking over all SRs, no significant excess of data over background is visible, no discovery of a signal can be claimed.

Since no excess has been found, the degree to which the models of interest can be excluded is evaluated. This statistical interpretation is performed in two ways:

- Model-Dependent Interpretation: For every set of parameters-here, either $\left(m(\tilde{g}), m\left(\tilde{\chi}_{1}^{0}\right)\right.$ or $\left(m(\tilde{q}), m\left(\tilde{\chi}_{1}^{0}\right)\right.$ — the corresponding CL of a hypothesis test of background-only versus signal+background hypothesis with respect to data is calculated. In a next step, a contour line is drawn along the values of $\mathrm{CL}=95 \%$ across the two-dimensional parameter plane. Since in every hypothesis test a certain signal model and its prediction in the studied SR is considered, such a set of results is model-dependent.

- Model-Independent Interpretation: In order to quantify how sensitive the performed analysis is towards finding any non-background signal, a model-independent interpretation is performed as well. In another hypothesis test the background-only hypothesis is tested against the observed data. The resulting level of (dis)agreement can be used to calculate upper limits on the production cross section of any model that is still compatible with the observed results.

Figures 4(a) and 4(b) show model-dependent results for two of the seven Simplified Models studied in this analysis, combining the most powerful results ${ }^{4}$.

\footnotetext{
${ }^{4}$ For the best combination, the result from the signal region with the lowest value of $\mathrm{CL}_{\mathrm{S}}$ is taken into account - this is equivalent to taking the SR with the strongest exclusion.
} 


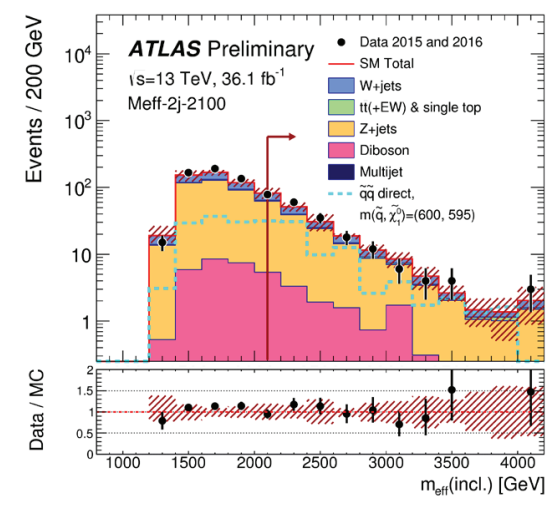

(a)

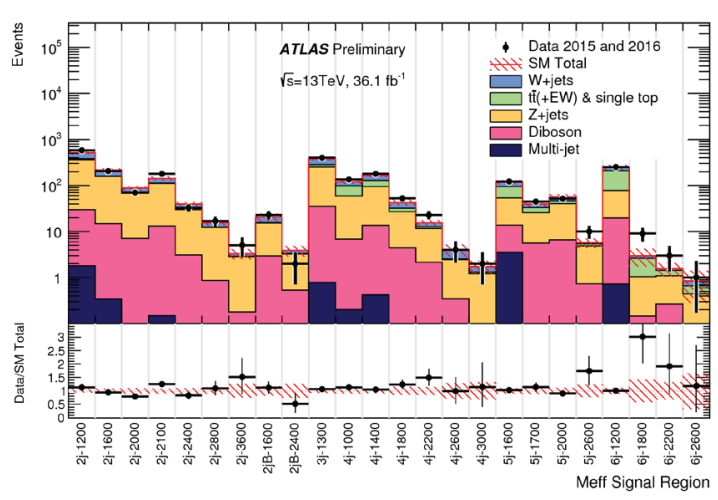

(b)
Distribution of the main discriminating variable $m_{\text {eff }}$ before the application of the final selection criterion on it and before application of the fitted background normalisation. The cut value is again indicated by the red arrow. The growth of the signal contribution with respect to predicted background is visible. The red line and hatched band again represent the total Standard Model expectation and its combined uncertainty (one standard deviation of combined statistical and systematic uncertainty). The last bin contains the overflow bin of the histogram. The lower panel represents the ratio data/MC with accordingly propagated uncertainties [4].
Summary of the agreement between observed data and the Standard Model expectation across all SRs after the fitted background normalisation is applied. While the upper panel shows the stacked Standard Model contributions and absolute yields, the lower panel shows the agreement as a ratio between data and simulation with accordingly propagated uncertainties [4].

Figure 3. Results in the signal regions of [4], (a) for an exemplary SR, (b) as an overview over all SRs.

In addition to the Simplified Models introduced in the beginning of section 4, a complete model of SUSY as introduced in section 2 is studied: the phenomenological MSSM (pMSSM) [9]. The 19 free parameters that describe the pMSSM are reduced to two - the mass of the gluino and the LSP_by imposing further assumptions. In a similar fashion as for the Simplified Model, the results are visualised in Figure 4(c) and 4(d): in this representation, the parameter plane is defined by the masses of the squark and the gluino while the mass of the LSP is fixed (massless case in Figure 4(c), massive case in Figure 4(d)). The case of the massless LSP reproduces the limit of the Simplified Model at low LSP masses and serves as a successful consistency check.

The model independent upper limits on production cross sections per signal region and breakdowns of the background contributions and systematic uncertainties can be found in [4].

\section{The Search for a Top Superpartner - Two Leptons as an Indicator for the Presence of the Light Top Squark}

As mentioned in section 3, the second major set of SUSY searches within the ATLAS collaboration is targeting third generation sfermions directly. One of the analyses driving the progress in the search for the light top squark is looking for its pair production and decays leading to the presence of Standard Model leptons [5]. Similar to the search presented in section 4, multiple Simplified Models are looked for. However, in this analysis, the motivation to study several models all at once is a different one: due to the fact that the light top squark is expected to be the Next-To-Lightest-SupersymmetricParticle (NLSP) in many models [1], it can be similar in mass to its Standard Model partner, the top quark, or much heavier. In order to account for the wide range of potential top squark masses and 


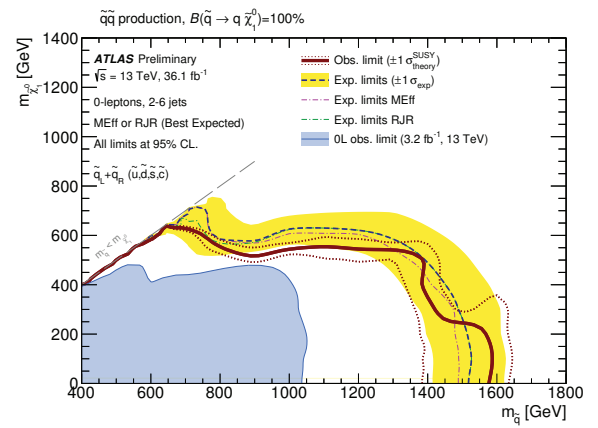

(a) Simplified Model, $\left(m(\tilde{q})-m\left(\tilde{\chi}_{1}^{0}\right)\right)$-plane

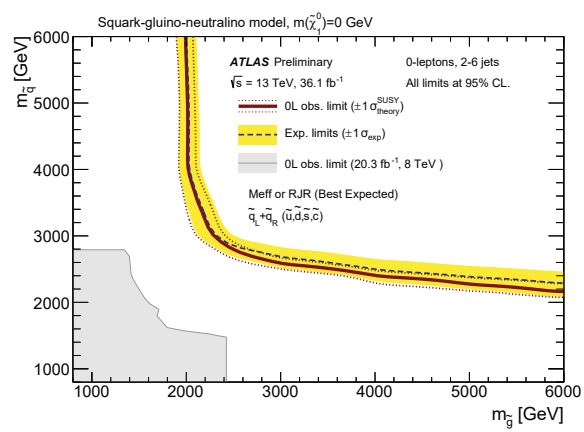

(c) pMSSM, massless LSP

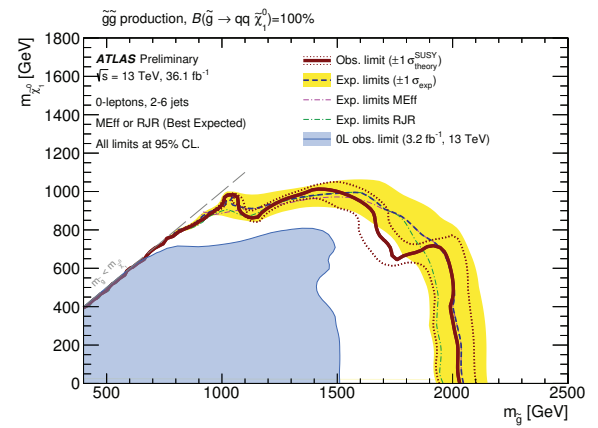

(b) Simplified Model, $\left(m(\tilde{g})-m\left(\tilde{\chi}_{1}^{0}\right)\right)$-plane

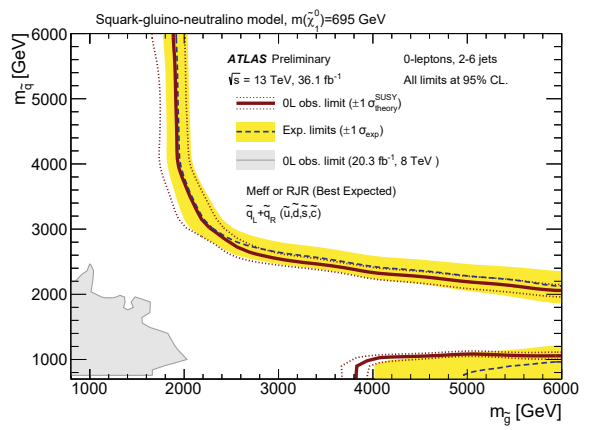

(d) pMSSM, massive LSP

Figure 4. Exclusion contours at $95 \%$ CL computed for different signal models in their corresponding space of free parameters. The dashed blue line and the solid yellow band give the expected limit and its one-standarddeviation combined uncertainty. The corresponding observed results are visualised by the solid red line and the dotted contour around it. (a) and (b) show the results of the Simplified Models of squark production (a) and gluino production (b) plotted in the mass-plane of the corresponding particle produced in the collision and the LSP. Here, the blue solid area marks the exclusion obtained in an earlier iteration of this analysis. (c) and (d) show the findings interpreted in an constrained version of the pMSSM where all parameters but the squark, gluino and LSP masses are fixed. The results are given in the $(m(\tilde{g})-m(\tilde{q}))$-plane. In (c) the LSP is set to be massless while (d) shows the limit with a massive LSP. The result of the massless LSP case in (c) reproduces the corresponding Simplified Model result shown in (b), serving as a sanity check for the consistency between the models [4].

their difference to Standard Model particles, different Simplified Models are proposed as depicted in Figure 5.

The Simplified Models cover the different mass hypotheses in the following way: the more particles participate in the decay of the top squark, the smaller the mass difference to its Standard Model partner. When the splitting between the two masses is large, enough energy is available to produce even another SUSY particle such as the lightest chargino (a) while a smaller difference could still allow for a decay into a top quark and a massive LSP (b). Compressing the mass spectrum further leads to the direct flavour changing decay into a $b$ quark and a $W$ boson at an effective vertex (c) while at lowest splittings the decay vertex is effective with the stop decaying into a $b$ quark and a lepton-neutrino pair directly.

The strategy of this study is similar to what has been presented in section 4.1: for each dominant Standard Model background, a designated control region is designed. A simultaneous fit of the back- 


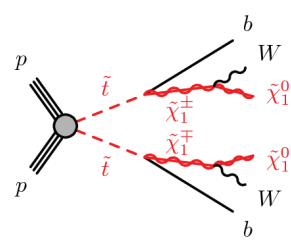

(a) 2-body decay, intermediate $\chi_{1}^{ \pm}$

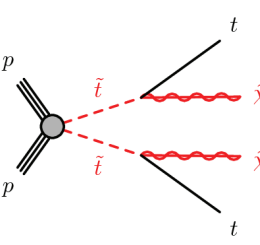

and

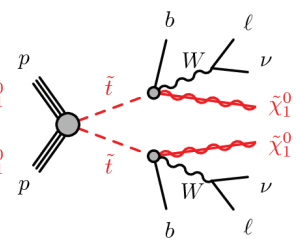

(c) 3-body decay, effective vertex

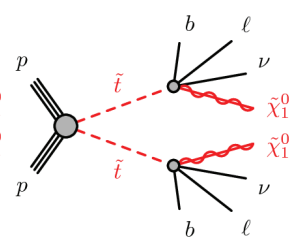

(d) 4-body decay, effective vertex

Figure 5. Signal diagrams of the Simplified Models as in [5]. Depending on the mass difference between the produced top squark and the Standard Model top quark, the decay process changes. The mass difference decreases from (a) to (d) prohibiting the decay to heavy particles such as a chargino (a), the top quark (b), or a real $W$-boson (c) until only light particles are produced (d) - the latter two cases make use of effective vertices.

grounds to the observed data in all CRs determines the normalisation of the Standard Model expectation to obtain a reliable prediction in the SRs. Exemplary control region distributions illustrating the agreement between the MC-based background predictions and data are shown in Figures 6(a) to 6(c). The observed agreement is good and can hence serve as a reliable prediction of the Standard Model backgrounds in the signal regions of interest.

Apart from the MC-based backgrounds, the most dominant background arises from fake and nonprompt leptons. This contribution is estimated in a data-driven way, described and validated in [5].

\subsection{Analysis Results \& Interpretations}

The background fit results obtained in the CRs are applied in the SRs. Distributions of variables used to define an exemplary SR are shown in Figure 6(d) and 6(e). Again, the arrow indicates the final signal region that is obtained when cutting accordingly.

From the observed data and the predicted background and signals, again, model-dependent and model-independent limits can be computed in the same fashion as outlined in section 5.1. The modeldependent results for the different Simplified Models are visualised in the $\left(m(\tilde{t})_{1}, m\left(\tilde{\chi}_{1}^{0}\right)\right)$-plane for all types of decays in Figure 7(a). The plot illustrates the power of this analysis: the gaps that were present after Run-I of the LHC (blue limit contours) are filled up and the overall picture is more completed - in addition to the general extension of the limit.

Similar to the analysis presented in section 4, this study, too, performs an interpretation of its results not only in the Simplified Models used for the search but also in the pMSSM. This analysis leaves one more degree of freedom and presents results for both possible signs of the Higgs mass parameter $\mu$ in the $\left(m\left(\tilde{t}_{1}, m\left(\tilde{\chi}_{1}^{0}\right)\right)\right.$-plane fixing the rest of the 16 model parameters as described in [5]. The obtained result is depicted in Figure 7(b). 


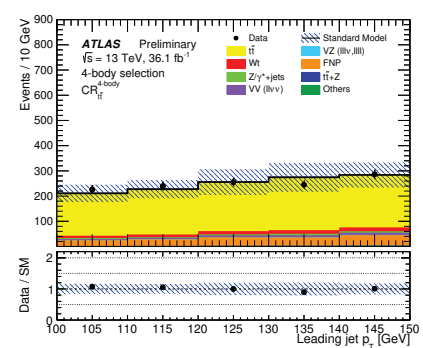

(a) $t \bar{t}$ 4-body-CR

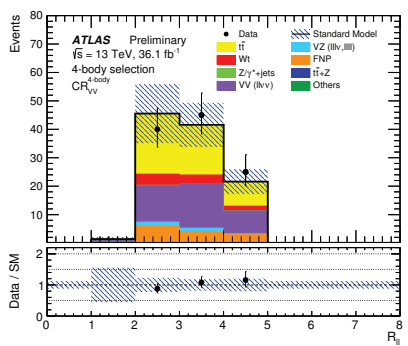

(b) $V V$ 4-body-CR

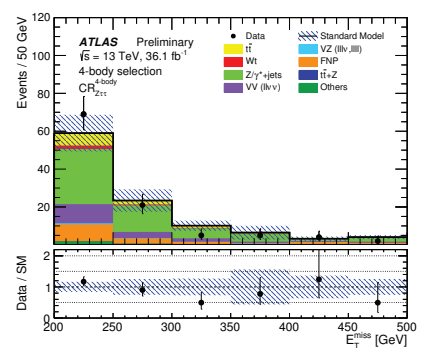

(c) $Z \rightarrow \tau \tau$ 4-body-CR

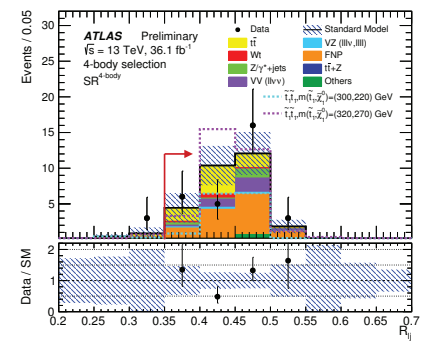

(d) $R_{l j}$, 4-body-SR

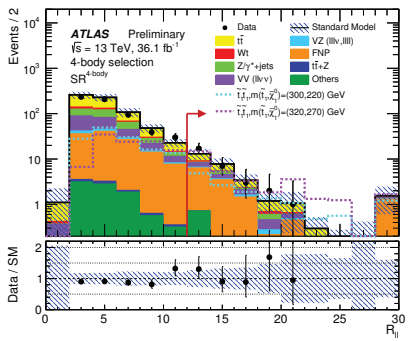

(e) $R_{l l}, 4$-body-SR

Figure 6. Exemplary kinematic distributions after the fit of the background normalisations in the CRs. The black line and its blue hatched band represent the total Standard Model expectation and its combined uncertainty (one standard deviation combination of statistical and systematic uncertainties). (a) to (c) show the control regions associated to the 4-body Simplified Model and its SR. Each plot depicts the distribution of a variable characteristic for the CR. (d) and (e) show distributions of the variables $R_{l j}$ and $R_{l l}$ that characterise the 4-body SR before their application (definitions in [5]). Again, the red arrows illustrate the selection criterion which is imposed to obtain the final SR selection. Here, a gain in signal contributions with respect to background is visible. Again, the lower panels show the bin-wise ratio of data/MC with the corresponding uncertainties. The agreement between prediction and observation in the CRs is visible. The last bin in each distribution contains the overflow bin of the histogram [5].

\section{Outlook \& Conclusions}

With two of the major analyses in the search program for strong production of SUSY within the ATLAS collaboration being described in detail, a brief look on even more specific analyses provides an idea of the full ATLAS scope on that topic.

While both analyses presented so far considered only R-parity conserving (RPC) models of SUSY, several studies searching for R-parity violating (RPV) SUSY are carried out, too. As an example the analysis targeting signatures of one lepton and multiple jets studying models of RPV SUSY can be considered [10]. Moreover, analyses using tau leptons such as [11] target the strong production of (RPC) SUSY. While all of the aforementioned studies consider only short-lived SUSY particles such as immediately decaying gluinos, few analyses try to look for longer-lived sparticles such as [12] that is able to probe areas of phase space which are not accessible using the more common signatures.

Concluding, a brief overview of the ATLAS collaboration's rich search program for strong production of Supersymmetry has been provided. The focus has been put on two of the main progress-driving analyses, their search strategies, the obtained results and their interpretation. Apart from the studies presented in detail, far more analyses address the topic of this work. Beyond the results compiled here, many analyses are still ongoing, relying on more recorded data due to rare signatures or better 


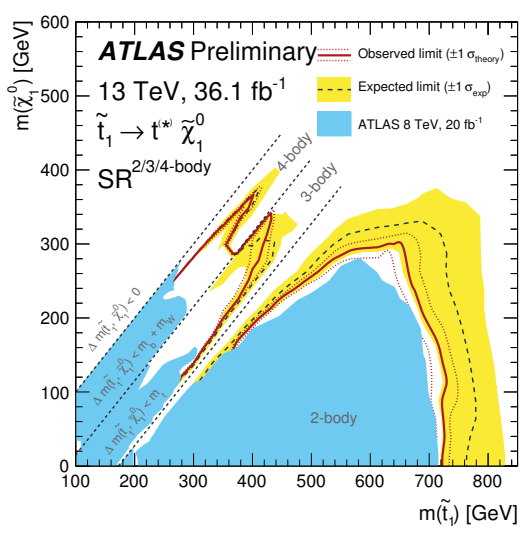

(a) Simplified Model

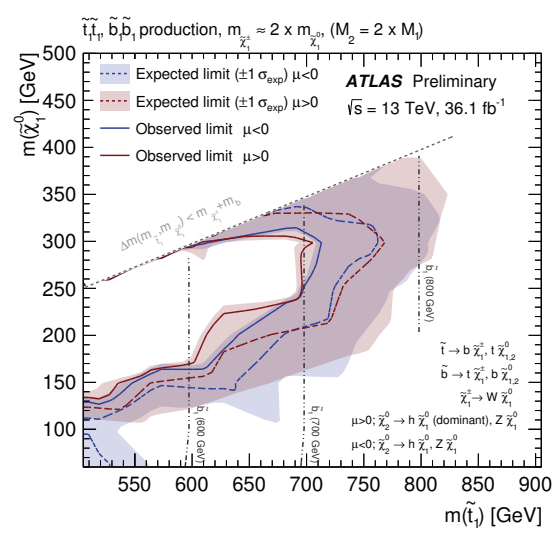

(b) pMSSM

Figure 7. Exclusion contours at $95 \%$ CL computed for the combination of all Simplified Models (a) and an interpretation in the pMSSM phase space (b). The dashed line and the solid band give the expected limit and its one-standard-deviation combined uncertainty. The corresponding observed results are visualised by the solid line and the dotted contour around it (not computed in (b)). (a) displays the results of the Simplified Model findings in the $\left.m(\tilde{t})_{1}\right)-m\left(\tilde{\chi}_{1}^{0}\right)$-plane. With the blue area depicting the Run-1 result of this search, both a filling of gaps in this plane and an extension of the exlcusion is visible. The still open gaps mark particularly difficult to access regions of phase space where the kinematics of the involved particles are hard to detect. (b) visualises the interpretation of the obtained results in the pMSSM model. In this interpretation the LSP mass and the top squark mass are left as floating parameters while all other parameters except the Higss mass parameter $\mu$ are fixed. Two interpretations for different values of $\mu$ are shown in red and blue, respectively. In addition, iso-lines for the light bottom squark are drawn to provide additional information for model builders and further interpretation [5].

calibrated data due to high sensitivity to the smallest of detector effects. With this in mind, ATLAS search for Supersymmetry continues and remains exciting.

\section{References}

[1] S.P. Martin (1997), adv. Ser. Direct. High Energy Phys.18,1(1998)

[2] ATLAS Collaboration, JINST 3, S08003 (2008)

[3] L. Evans, P. Bryant, JINST 3, S08001 (2008)

[4] ATLAS Collaboration, ATLAS-CONF-2017-022, https://cds.cern.ch/record/2258145

[5] ATLAS Collaboration, arXiv:1708.03247[hep-ex]

[6] CMS Collaboration, Phys. Rev. D96, 032003 (2017)

[7] CMS Collaboration, arXiv:1705.04650[hep-ex]

[8] CMS Collaboration, CMS-PAS-SUS-17-001, http://cds . cern. ch/record/2256753

[9] ATLAS Collaboration, JHEP 10, 134 (2015)

[10] ATLAS Collaboration, arXiv:1704.08493[hep-ex]

[11] ATLAS Collaboration, Eur. Phys. J. C 76, 683 (2016)

[12] ATLAS Collaboration, Phys. Rev. D 93, 112015 (2016) 